\title{
EVALUATION OF MINI-LOCKING PLATE SYSTEM IN MANAGEMENT OF BODY MANDIBULAR FRACTURE (CLINICAL STUDY)
}

\author{
Mohamed M Ghoniem*, Bahaa El-Din A Tawfik**
}

\begin{abstract}
Aim: To evaluate the mini-locking plate system in management of body mandibular fracture. Patient and methods: Twenty patients were randomly classified into two equal groups. Group (1): (The study group): Ten patients were treated by open reduction and internal fixation using single $2.0 \mathrm{~mm}$ locking plate and arch bar as a tension band maintained for 5 weeks. Group (2): (The control group) Ten patients received the same treatment as in group (1) but with single $2.0 \mathrm{~mm}$ conventional plate. Follow- up was performed for 6 months and post-operative complications such as infection, wound dehiscence, neurosensory disturbances and malocclusion were recorded. Arch bar was removed after 5 weeks of surgery. Data regarding age, sex, and bone density at the fracture side during healing, bite force measurements at anterior, premolar and molar regions were collected, tabulated and statistically analyzed using IBM SPSS software package version 20.0.
\end{abstract}

Results: Uneventful healing of all fractures treated with mini-locking plates and conventional plate system, with no cases of malunion, nonunion, infection, or malocclusion being observed through the follow up periods. There was statistically difference between both groups regarding the mean bone density one week, 1,3 and 6 months post-operatively. Bite force recording showed increasing values at subsequent follow ups corresponding to the healing of the fracture in both groups.

Conclusion: Bone density in group (1) was in an accelerated pattern and of higher value than that of group (2). Patients treated with locking plate screw system regain more bite force compared to those treated with conventional miniplate screw system.

\section{INTRODUCTION}

Mandibular fracture is the first or second most common facial bone fracture, occurring as twice as fractures of the midface bones. The incidence is about $38 \%$ of all facial bone fractures. This is mainly due to mandibular prominence, relative lack of support, and the weak structures of the condyles, angles and parasymphyseal regions of mandible ${ }^{(1-2)}$.

Successful treatment of mandibular fractures depends on reduction and fixation for restoration of the normal occlusion, function and proper alignment of bone using a closed or open technique ${ }^{(3)}$. Current management of mandibular fractures consists of early surgical intervention with exposure of the fracture and placement of rigid fixation. Internal rigid fixation for mandibular fracture has been recognized as a reliable method for obtaining osteosynthesis. It may allow for early active physiotherapy and resumption of normal function ${ }^{(4)}$. Various types of bone plating systems have been developed to provide stable fixation for mandibular fractures. A disadvantage of conventional bone plate/screw system is that the plate must be perfectly adapted to the underlying bone to prevent alterations in the alignment of the segment and changes in the occlusal relationship. The introduction of locking plate/screw plating systems for the treatment of mandibular fracture has offered certain advantages over other plating systems ${ }^{(5,6)}$.

In the locking plate system, the screw locks not only to the bone but to the bone plate.

* Lecturer of Oral and Maxillofacial Surgery, Faculty of Dental Medicine (Sinai University)

** Ass. Professor of Oral and Maxillofacial Surgery, Faculty of Dental Medicine (Boys- Cairo, Al-Azhar University). 
This is accomplished by having a screw with a double thread, one will engage the bone; another will engage a threaded area of the bone plate. The result is a locking plate system which in effect provides a mini-internal fixator. since the plate locks to the screw rather than gaining its rigidity by being compressed against the bone, it also avoids the cortical necrosis which sometimes seen under a plate which is compressed against the bone. ${ }^{(7)}$

Minilocking plates: Herford and Ellis concluded that the use of a locking plate screw system was simple, and it offers advantages over conventional bone plates by not requiring the plate to be compressed to the bone to provide the stability ${ }^{(8)}$. Moreover, many studies have shown improved the stability of this system in compared with the nonlocking miniplates, especially in decreasing the torsion and the opening of the fracture site ${ }^{(9)}$. Alpert demonstrated that the locking system between the screw and the plate which act as internal fixator. This locking mechanism was used in orthopedic and neurosurgery before several years and the results of this system was satisfying in providing more stability with minimal complication ${ }^{(10)}$. On the other hand, the experimental studies showed the mechanical and biological advantages on locking plates, which allow for more rapid and undisturbed bone healing as well as decreased risk of delayed union, nonunion or infection ${ }^{(11)}$.

Bite force measurements: Bite force is one indicator of the functional status of the masticatory system that results from the action of jaw elevator muscles modified by the craniomandibular biomechanics. Since the biting force is an important parameter in assessing the masticatory function, the aim of this study was to evaluate the maximum bite force in patients with body mandibular fractures treated with minilocking plate and conventional plate and also to determine the rate of recovery of the bite force ${ }^{(12)}$.

\section{PATIENTS AND METHODS}

Patients were selected from those attending outpatient clinics of Oral and Maxillofacial surgery department of Saied Galal Hospital, Faculty of Dental Medicine, Al-Azhar University. The selected patients would have non-comminuted mandibular body fracture excluding the condyle and coronoid process and having a dentition enough to apply arch bars. Their ages ranged from 20 to 40 years, mean age 32.5 years. All patients will sign a written consent after being informed about the procedures, prognosis and complications of the surgery. Twenty patients were randomly classified into two equal groups each one comprises ten patients. Group1: The selected cases will be treated by open reduction and internal fixation using single $2.0 \mathrm{~mm}$ locking plate and additional arch bar placed as a tension band maintained for 5 weeks. Group 2: A similar number of patients received the same treatment as in Group (1) but with single $2.0 \mathrm{~mm}$ conventional plate. Patients were admitted to the hospital and scheduled for the operation. All laboratory investigations required for general anesthesia was done. Patients underwent general anesthesia via naso-tracheal intubation. The arch bar was placed for upper and lower jaw followed by inter maxillary fixation. A lower vestibular incision was made in the labiobuccal sulcus and a mucoperiosteal flap was raised to expose the fracture line till the lower border of the mandible. The entrapped tissue between the fractured segments was curetted using a small surgical curette; avoid damage to the mental nerve.

Precise reduction, plate fixation, Trans- buccal technique was used if needed for fixation of the plate and screws and wound was closed by stitching with 3/0 Vicryl sutures. MMF was opened, occlusion was verified. Oral antibiotics were prescribed postoperatively for 5 days, soft diet for 4 weeks.

Postoperative evaluation was performed clinically at 5 weeks, 3 and 6 months. Many clinical parameters where be assessed for each patient 
at each follow up visit, pain, swelling, infection, wound dehiscence, neurosensory disturbance and malocclusion were recorded. Arch bar was removed after 5 weeks of the surgery.

Radiographic evaluation was being done with digital panoramic radiograph first postoperative day, one week, 1, 3 and 6 months postoperatively. Radiographic analysis was performed both qualitatively and quantitively (Radio densitometric analysis), Fig. (1-2)

Radiometric data are used to determine relative changes in the bone density by quantifying the mean grey values of image either through density changes through lines or by determining the region of interest (ROI) ${ }^{(13)}$. The term density refers to the degree of whiteness of the radiographic image which consists of points, or pixels, each having certain density value. Bone density changes along the fracture line were performed on all base line and follow up images of the study and control groups. Using the same program, linear density measurements were performed by drawing lines parallel to each other covering the fracture line. Density changes along each of these lines were calculated and their mean was recorded to present the density changes, Fig (3).

Bite force measurement device: The device was designed and composed of:

1. Load cell: that converts the load into electrical signal.

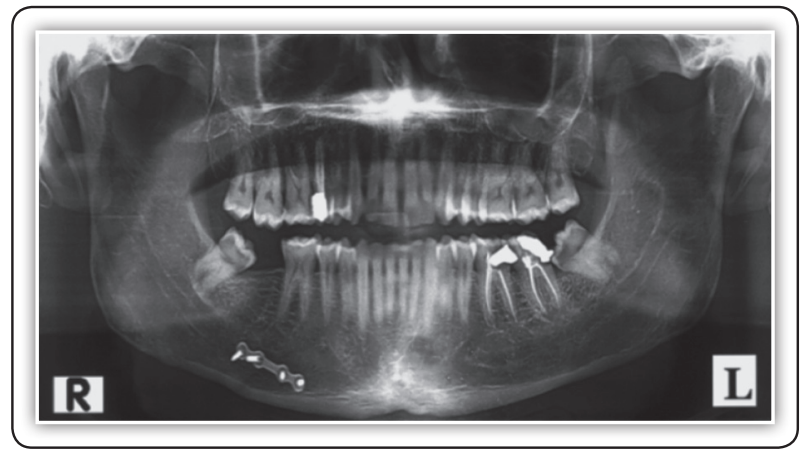

FIG (1) Panoramic view showing mandibular fracture at the right-side Group (1)
2. The metal fork: this fork was drawn on paper and designed as a metal one by the lather at the turnery.

3. Amplifier: which magnify the electrical signal and transform it to the indicator.

4. Digital indicator: Once the output signal is amplified, one can calibrate the load cell and the collected data was registered.

Procedure: $\cdot$ Select the appropriate size of mouth prob which was autoclaved.

- Cover the metallic fork with gloves.

- Power on the device.

- Put the mouth prob between the upper and lower teeth, Fig (4)

- Each patient was seated with the head upright and in unsupported natural position so that the Frankfort horizontal plane would be parallel to the floor. At the beginning of the test, each subject was asked to bite on the mouth prob in order to make him familiar with the equipment and no measurements were made. After that a series of three consecutive recordings were taken and noted. The rest period of one minute was given between each recording to prevent muscle fatigue. Mean of the three recordings was taken as the maximum bite force. The patient was instructed to bite on the mouth prob as hard as possible for 5 seconds at the central area, right molar area and left molar area.

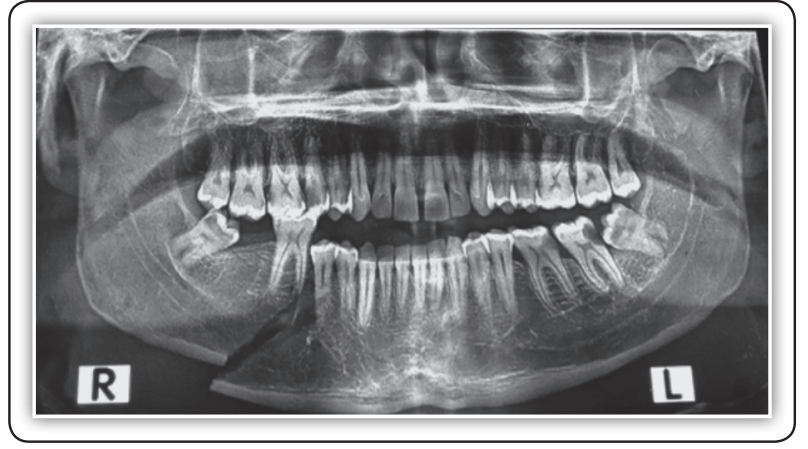

FIG (2) Showing six-month Postoperative Group (1) 


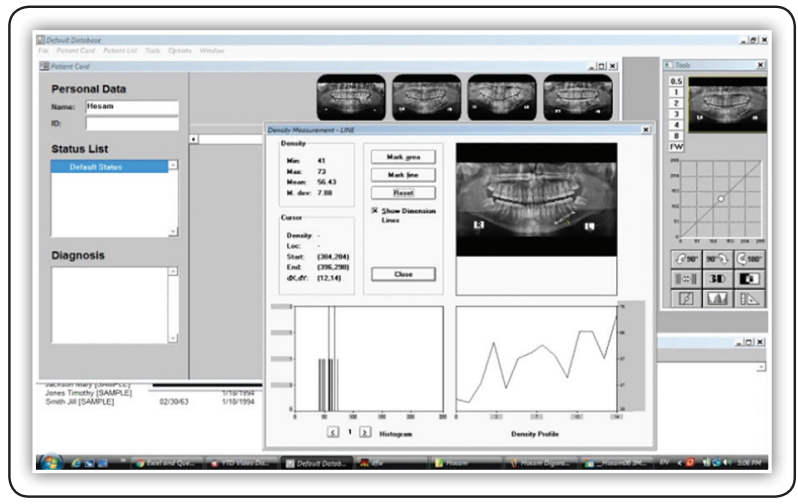

FIG (3) Showing bone density measurement at 6 months Group (2)

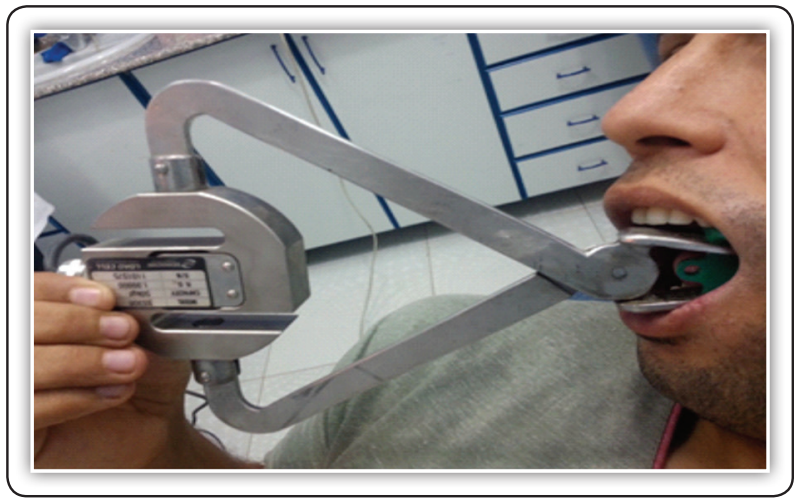

FIG (4) Showing bite force measurement

Data were collected, tabulated and statistically analyzed using IBM SPSS software package version 20.0 .

Chi-square test was used to analyze qualitative data (which were described as number and percent). Quantitative data were described as mean and standard deviation. Independent student "t-test" was used for comparison of selected parameters of both groups in at the same interval, while One-Way ANOVA was used to compare between different intervals in each group. Results were considered statistically significant at $\mathrm{p}<0.05$.

\section{RESULTS}

In both groups the pain score decreased and became significant at the end of 6 weeks and after 3 months post-operatively. There was statistically significant difference regarding Group (1) after 6 weeks and after 3 months $(\mathrm{P} 1<0.05)$. There was statistically significant difference regarding Group (2) after 6 weeks and after 3 months (P1<0.05). None of the patients in the locking group while, $10 \%$ in the control group developed infection which was managed with antibiotics. During follow up, all 5 patients still complained of residual sensory impairment which showed gradual improvement till the third month post-operatively where normal sensation was restored.In Group (1),90\% of patients had satisfactory occlusion, while only $10 \%$ of patients presented with mild derangement. In Group (2), $70 \%$ of the patients had satisfactory occlusion, while $30 \%$ had occlusal derangement which then treated with elastics. On $1^{\text {st }}$ and $3^{\text {rd }}$ month follow up, all patients $(100 \%)$ presented with satisfactory occlusion. There was no statistically significant difference between the two groups regarding the sensory function, surgical wound or occlusion.

In Group (1) there was statistically significant increase in bone density after one, three and six months postoperatively. In Group (2) at six months the increase in bone density was statistically significant. Comparing both groups at different follow up periods, bone density of group (1) was higher than that of group (2) in all follow up periods, Fig. (5), Table (1).

Bite Force Measurements: The bite force was measured using the designed apparatus. The bite force measurement was conducted following the surgical operation at 1 week, 2 weeks, 3 weeks, 4 weeks, 5 weeks, 6 weeks and 12 weeks. The bite force was measured at the anterior teeth, premolar region (right and left) and at molar region (right and left).

Bite force measurements at right molar region: In both groups, the biting force in the right molar region increased significantly after 2 weeks till the end of the follow up intervals in both groups. Comparing both groups, there was no statistically significant difference till the 3rd week postoperatively. At the 4th and 5th weeks postoperative intervals the biting force at the right molar region of group (1) was statistically lower than of group (2). There was no statistically 
difference at week 6 postoperative interval. At 12th week the biting force at the right molar region of group (1) was statistically lower than of group (2), Table (2), Fig (6).

Bite force measurements at left molar region: In both groups the biting force in the left molar region increased significantly after 2 weeks till the end of the follow up intervals in both groups. Comparing both groups, there was no statistically significant difference at $1^{\text {st }}$ and $2^{\text {nd }}$ weeks postoperative. Starting from the 3 rd week postoperative interval the biting force at the left molar region of group (1) was statistically lower than of group (2). There was no statistically significant difference at the 6th week postoperatively. At 12th week the biting force at the left molar region of group (1) was statistically higher than of group (2).

Table (1): Comparison between the two studied groups according to bone density at different periods of follow up.

\begin{tabular}{|c|c|c|c|c|}
\hline $\mathbf{P}$ & "'t"' & Group II & Group I & \\
\hline $0.172 \mathrm{NS}$ & 1.424 & $\begin{array}{c}34.7-94.4 \\
56.11 \\
22.51\end{array}$ & $\begin{array}{c}51.7-97.9 \\
68.49 \\
15.78\end{array}$ & $\begin{array}{c}\text { Post-operative } \\
\text { - Range } \\
\text { - Mean } \\
\text { - S.D. }\end{array}$ \\
\hline $0.103 \mathrm{NS}$ & 1.717 & $\begin{array}{c}39.8-101.2 \\
63.61 \\
22.65 \\
\end{array}$ & \begin{tabular}{|c|}
$52.12-119.5$ \\
79.85 \\
19.53 \\
\end{tabular} & \begin{tabular}{|c|}
1 week \\
- Range \\
- Mean \\
- S.D.
\end{tabular} \\
\hline $0.065 \mathrm{NS}$ & 1.976 & $\begin{array}{c}42.6-119 \\
70.04 \\
24.86 \\
\end{array}$ & $\begin{array}{c}75-140.4 \\
89.77 \\
19.70 \\
\end{array}$ & $\begin{array}{c}1 \text { month } \\
\quad \text { Range } \\
\text { - Mean } \\
\text { - S.D. }\end{array}$ \\
\hline $0.070 \mathrm{NS}$ & 1.925 & $\begin{array}{c}45.3-119.5 \\
77.64 \\
23.97 \\
\end{array}$ & $\begin{array}{c}79.3-154 \\
97.49 \\
22.10 \\
\end{array}$ & $\begin{array}{c}3 \text { months } \\
\text { - Range } \\
\text { - Mean } \\
\text { - S.D. }\end{array}$ \\
\hline $0.077 \mathrm{NS}$ & 1.879 & $\begin{array}{c}52.6-138.9 \\
86.60 \\
26.67 \\
\end{array}$ & \begin{tabular}{|c|}
$85.6-165.5$ \\
107.87 \\
23.87 \\
\end{tabular} & $\begin{array}{c}6 \text { months } \\
\cdot \text { Range } \\
\cdot \text { Mean } \\
\cdot \text { S.D. } \\
\end{array}$ \\
\hline & & 2.414 & 5.614 & $F$ ratio \\
\hline & & $0.063 \mathrm{NS}$ & $0.001 *$ & Probability \\
\hline & & 21.77 & 18.356 & $L S D$ \\
\hline
\end{tabular}

NS: no significant difference

*: significant difference

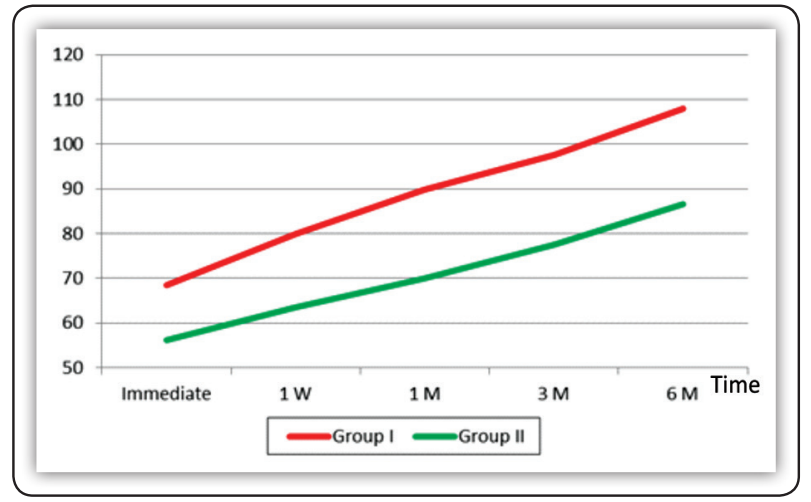

FIG (5) Diagram showing the comparison between the two groups regarding bone density at different period of follow up.

TABLE (2): Comparison between the two studied groups regarding the bite force at right molar area at different periods of follow up.

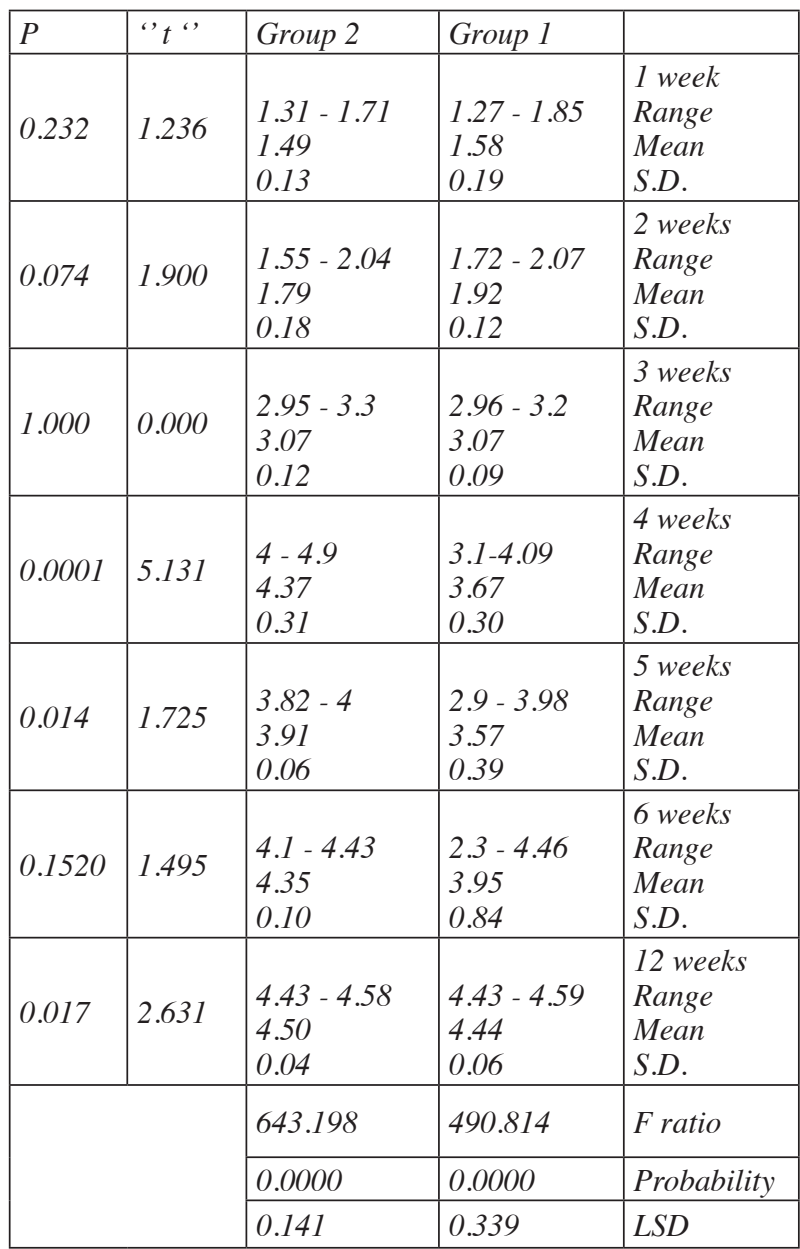




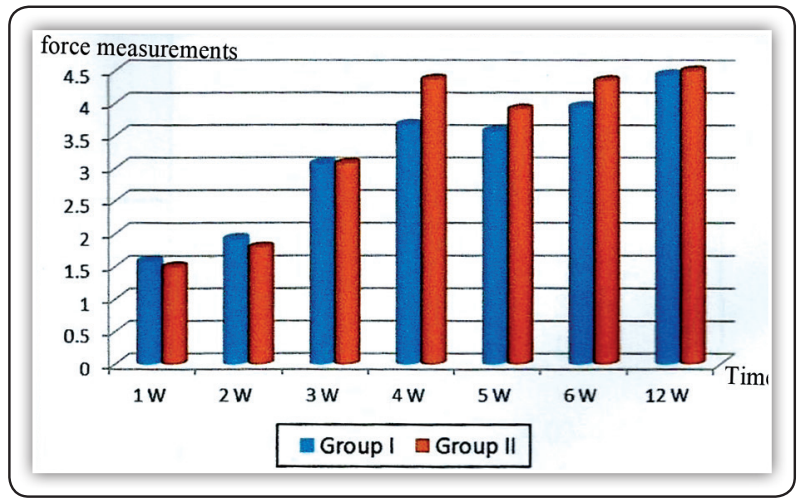

FIG (6) Bar chart showings mean values of bite force measurements at right molar region.

\section{DISCUSSION}

In the present study no, postoperative infection has been observed, though two patients presented with preoperative infection. This was in agreement with Gerlak et al (14) as no infection has been developed along the study follow up periods as they used the miniplates osteosynthesis and regard to the teeth within the line of the fracture was firmly retained. In the present study, single miniplate was used in the compression area of fracture accompanied by the arch bar placed as a tension band maintained for 5 weeks for its superior advantages including; maximize the advantages of an ORIF technique, more reliable, minimized implanted material, cost effective and decreasing postoperative complications without affecting the stability of the fractured segments as reported by several researchers ${ }^{(15-16)}$. It is difficult to calculate the extent to which a reduction of the total amount of titanium used will decrease deposition of metal ions in the peripheral organs. Nevertheless, it should be the foremost aim to use as little osteosynthesis material as possible ${ }^{(17)}$.

Pain intensity was significantly decreased in both groups across the follow up period which may be attributed to adequate stabilization of the fracture segments. This was in accordance with Manoj Goyal et al ${ }^{(18)}$, they mentioned that the edema score postoperatively was higher in the conventional group than in the locking group which may indicate less traumatic manipulation of the soft tissue during placement of the plates. The maximal mouth opening was inadequate in both groups immediately postoperative, while increased gradually in the study group on the 2 nd week which was associated with noticeable decrease in pain and edema postoperatively in both groups, this was in alignment with Singh V et al ${ }^{(19)}$, they mentioned that the trismus decreased gradually in the locking group postoperatively, while it increased in the non-locking group postoperatively. The current study reported 5 cases with sensory impairment in the lower lip; one of them was reported postoperatively in the control group with no signs of this impairment preoperatively. This sensory impairment could be attributed to fracture manipulation, rather than drilling and screws placement close to the nerve as reported by Guimond et al ${ }^{(20)}$.

All cases of the current study started to regain their normal sensation gradually by the end of 12 weeks postoperative follow up. On the other hand, this sensory disturbance didn't affect percentage in bite forces in comparison to the other cases without sensory impairment. In the present study no, postoperative malocclusion was observed and this may be regarded to the rigidity of minilocking plate so no need for MMF and this was in agreement with Ellis et al recommendations ${ }^{(21)}$. Also, there was no need for placement of a second plate and this was in agreement with Scolozi et al ${ }^{(15)}$ they concluded that a single $2.0 \mathrm{~mm}$ locking plate is associated with no major complications and sound bone healing in all patients. Clinical examination of both groups showed the stability of the fracture segments as well as proper occlusion except in one case in the control group which was treated by elastic traction for two weeks and hence the occlusion was good and this may be regarded to the low rigidity of conventional plate, the trials for adaptation of the plate reduced its rigidity or due to the defect in arch bar application. Radiographic interpretation of the bone density had been proven to be one of the most valuable means to clarify healing progress. It had the advantages of being simply manipulated technique so, serial digital standardized orthopantograms were taken immediately, one week, 
one month, 3 months and 6 months postoperatively to detect and measure the changes in bone density using the Digora software which seemed to be more accurate and easier than other methods and this was in agreement with Bhupendra Harjani et $\mathrm{al}^{(22)}$ as the increase in the study group was in an accelerated pattern and of higher value than the control group. Locking plate screw systems offer certain advantages over other plates, where no need for precise adaptation to the underlying bones, otherwise the screw lock to the plate provides more stabilization, as the stability is achieved by locking the screw to the plate.

Moreover, during tightening the screw to the conventional plate, torsion as well as gapping between the bone fragments could occurred, while in case of locking plates system, fixation of the plates with locking screws kept the reduction nearly unchanged, therefore the plate does not have to be contoured as precisely as with conventional plating systems ${ }^{(21)}$. There are some advantages of the locking bone plating systems like that ; screws are unlikely to loosen from the bone plate, this means that even if a screw is inserted into the fracture gap, loosening of the screw will not occur. The possible advantage to this property of a locking plate screw system is a decreased incidence of inflammatory complication from loosening of the hardware and this was in agreement with Cordey et $\mathrm{al}^{(23)}$, they concluded that the friction between screw head and plate is the main weak point of the entire fixation, in the locking plate screw system, the thread on the screw head locks into the congruent thread of the plate, transforming the screw and plate into a unit creating a rigid splint with higher mechanical stability.

The present study evaluated the bite force measurements in patients whereas the healing was considered as dynamic process increasing gradually and observed through the masticatory force that was easy to be measured, as the bite force showed the highest values in molar regions followed by premolars and anterior teeth regions and this was in agreement with Tortopidis et $\mathrm{al}^{(24)}$, Shinogaya et $\mathrm{al}^{(25)}$ and Ferrario et al ${ }^{(26)}$. The reduction of bite force in the $1^{\text {st }}$ week postoperatively could be explained by the surgical trauma to the masseter muscle or by the protective neuromuscular mechanisms of the masticatory system as the muscle splinting components are activated or deactivated to prevent forces on the damaged bone, furthermore, the patients willingness to bite hard is also considered as a major factor.

The present study was in agreement with the study of Kumar ST et $\mathrm{al}^{(27)}$, they evaluated bite force in the incisor and molar region after open reduction and internal fixation using microplates, they founded that the bite forces increased gradually till reached the end of the follow up periods.

The significant dropping in the bite force values in the fifth postoperative week in both groups which was extended into the six weeks in the control group, may be due to the regeneration of the inferior alveolar nerve and innervation of the reflected periosteum with return of pain sensation which goes in accordance with Tate GS et al ${ }^{(28)}$.

The maximum bite force in healthy person at the left molar regions was $5.80 \mathrm{~N}$, at the right molar area was $6.79 \mathrm{~N}$, at the left premolars was $5.43 \mathrm{~N}$, at the right premolars was $5.65 \mathrm{~N}$ and at the anterior regions $3.91 \mathrm{~N}$, so the bite force showed increase steadily from $1.42 \mathrm{~N}$ to $2.39 \mathrm{~N}$ at the incisors area while right premolar region increasing from $1.63 \mathrm{~N}$ to $3.9 \mathrm{~N}$ and at left premolar region from $1.63 \mathrm{~N}$ to $3.8 \mathrm{~N}$, whereas at the right molar region from $1.85 \mathrm{~N}$ to $4.59 \mathrm{~N}$ and at the left molar region $1.81 \mathrm{~N}$ to $4.66 \mathrm{~N}$, this demonstrate that the rate of recovery of maximum bite force in treated mandibular fracture is steady even after 12 weeks period, but not returned to the normal range and this was in agreement with Kumar et al ${ }^{(29)}$.

\section{CONCLUSION}

Locking plate screw system provided adequate stability and reduces the amount of hardware with minimum chances of screws loosening and cortical necrosis Bone density in the study group was in an accelerated pattern and of higher value than the control group. 
Fracture treatment in the body mandibular region with one miniplate and arch bar for five weeks was effective to provide stability, early immobilization and optimal osteogenesis at fracture site, additionally decreasing complication, and cost.

\section{REFERENCES}

1. De Matos, F.P., F.M., Arenz and C.E. Trivellat: A retrospective study of mandibular fracture in a 40-month period. Int J Oral Maxillofac Surg.2010; 10, 39.

2. Alagoz, M.S., A.C. Uysal and O. Sensoz: An alternative method in mandibular fracture treatment: bone graft uses instead of a plate. J Cranio Fac Surg.2008; 19:411.

3. Coburn, D.G., D.W.G. Kennedy and S.C. Hodder: Complication with intermaxillary fixation screws in the management of fracture mandibles: $\mathrm{Br} \mathrm{J}$ Oral Maxillofac Surg.2002; 40:241.

4. Champy M, Lode J, Schmitt R: Mandibular osteosynthesis by miniature screwed plates via a buccal approach.J. Maxillofac Surg.1978; 6:14.

5. Kushner G, Alpert B: Management of mandibular body fractures. Atlas Oral Maxillofac Surg. Clin. North Am.1997; 5:61.

6. Raveh J, Roux M and Sutter F: The lingual application of a reconstruction plate: Anew method in bridging lower jaw defects. J. Oral Maxillofac Surg.1985; 43:735.

7. Soderholm A, Lindquist $\mathrm{C}$ and Skutnabb K: Bridging of mandibular defects with two different reconstruction systems: An experimental study. J. Oral Maxillofac surg. 1991; 49:1098.

8. Herford AS and Ellis E: Use of locking reconstruction plate and screw system for mandibular surgery. J Oral Maxillofac surg.1998; 56:1261-5.

9. Suerbier S, Schon R, Otten JE: The development of plate osteosynthesis for the treatment of fractures of the mandibular body- A literature review. J Craniomaxillofac Surg. 2008; 36:251.

10. Alpert B, Gutwald R, Schemlzeisen R. New innovations in craniomaxillofacial fixation: The 2.0 locking system. Keio J med.2003; 52:120-7.

11. Gardner MJ, Helfet DL, Lorich DG: Has locked plating completely replaced conventional plating? Am J Orthop.2004; 33:439.
12. Bakke M: Bite force and occlusion. Semin Orthod.2006; 12:120-126.

13. Lida S and Matsuya T: Pediatric maxillofacial fractures: Their etiologic characters and fracture patterns. J Craniomaxillofac Surg 2000; 19:60-4.

14. Gerlach KL, Schwarz A: Bite forces in patients after treatment of mandibular angle fractures with miniplate osteosynthesis according to Champy. Int J Oral Maxillofac Surg.2002; 31:345-348.

15. Scolozzi P, Martinez A and Jaques B: Treatment of linear mandibular fractures using a single 2.0-mm AO locking reconstruction plate: Is a second plate necessary? J Oral Maxillofac Surg.2009; 67:2636-2638.

16. Fernandez JR, Gallas M, Burgera M and Viano JM: A three dimensional numerical simulation of mandible fracture reduction with screwed miniplates. J Biomech.2003; 36(3):329-37

17. Rosenberg A, Gratz KW and Sailer HF: Should titanium miniplates be removed after bone healing is complete? Int J Oral Maxillofac Surg.1993; 22:185-188.

18. Manoj Goyal, Karan Marya, Sonia Chawla and Richa Pandey: Mandibular osteosynthesis: A comparative evaluation of two different fixation systems using $2.0 \mathrm{~mm}$ titanium miniplates and 3-D locking plates. J Maxillofac Oral Surg.2011; 10:316-20.

19. Singh V, Kumar I and Baghol A: Comparative evaluation of $2.0-\mathrm{mm}$ locking plate system vs $2.0 \mathrm{~mm}$ non-locking plate system for mandibular fracture: A prospective randomized study. Int J Oral Maxillofac Surg.2011; 40(4): 372-377.

20. Guimond C, Johnson J and Marchena J: Fixation of mandibular angle fracture with a $2.0 \mathrm{~mm} 3$-dimensional curved angle strut plate. J Oral Maxillofac Surg.2005; 63(2):209-14.

21. Ellis III E: Treatment methods for fractures of mandibular angle. Int J Oral Maxillofac Surg.1999; 28:243-52.

22. Bhupendra Harjani, Singh RK and Geeta Singh: Locking versus non-locking reconstruction plates in mandibular reconstruction. J Oral Maxillofac Surg.2012; 3:159-65.

23. Cordey J Perrens: Stress protection due to plates: Myth or Reality? A parametric analysis made by using the composite beam theory. Injury; 31(suppl 3), 2000.

24. Tortopids D, Lyons MF, Baxandale RH and Glimour WH: The variability of bite force measurements between sessions in different positions within the dental arch. J Oral Rehabil.1998;25:681-686. 
25. Shinogaya T, Bakke M and Thomsen CE: Effect of ethnicity, gender and age on clenching force and load distribution. Clin Oral Invest.2001; 5:63-68.

26. Ferrario VF, Sforza C, Zannoti G and Tartagilia GM. Maximal bite force in healthy young adults as predicted by surface electromyography.J Dent.2004; 32: 451- 457.

27. Kumar ST, Saraf S and Devi SP: Evaluation of bite force after open reduction and internal fixation using microplates. J Dent (Tehran).2013 10(5):466-477.
28. Tate GS, Ellis E III, Throckmorton G: Bite forces in patients treated for mandibular fracture-Implications fixation- Recommendations. J Oral Maxillofac Surg.1994; $52: 734$.

29. Kumar PK, Jayakumar K, Soumithran CS et al.: Comparative evaluation of bite force analytical study following mandibular osteosynthesis using three dimensional and conventional locking miniplates. National J of Maxillofacial Surgery.2017; 8: 34-40. 